\title{
Effects and safety of platelet-rich plasma (PRP) and hyaluronic acid (HA), alone and in combination, in the treatment of knee osteoarthritis: A systematic review and meta-analysis.
}

\section{Jinlong Zhao}

Guangzhou University of Chinese Medicine

Hetao Huang

Guangzhou University of Chinese Medicine

\section{Guihong Liang}

Guangzhou University of Chinese Medicine

\section{Ling-feng Zeng}

Guangzhou University of Chinese Medicine

\section{Weiyi Yang}

Guangzhou University of Chinese Medicine Jun Liu ( $\boldsymbol{\nabla}$ liujun.gdtcm@hotmail.com )

Department of Orthopaedics, Second Affiliated Hospital of Guangzhou University of Chinese

Medicine(Guangdong Provincial Hospital of Chinses Medicine) https://orcid.org/0000-0002-1943-3880

\section{Research article}

Keywords: Platelet-Rich Plasma, Hyaluronic Acid, Knee Osteoarthritis, Meta-analysis

Posted Date: February 11th, 2020

DOI: https://doi.org/10.21203/rs.2.23232/v1

License: (1) (i) This work is licensed under a Creative Commons Attribution 4.0 International License. Read Full License

Version of Record: A version of this preprint was published at BMC Musculoskeletal Disorders on April 11th, 2020. See the published version at https://doi.org/10.1186/s12891-020-03262-w. 


\section{Abstract}

Background Studies have shown that the combined application of hyaluronic acid (HA) and platelet-rich plasma (PRP) can repair degenerated cartilage and delay the progression of knee osteoarthritis (KOA). The purpose of this study was to explore the efficacy and safety of PRP combined with HA in the treatment of KOA compared with intra-articular injection of PRP or HA alone. Methods The PubMed, Cochrane Library, EMBASE and China National Knowledge Infrastructure (CNKI) databases were searched from inception to December 2019. Two orthopaedic surgeons conducted the literature retrieval and extracted the data. Outcome indicators include the Western Ontario and McMaster Universities Arthritis Index (WOMAC), the Lequesne Index, the visual analogue scale (VAS) for pain, and adverse events (AEs). Results Seven studies (5 randomized controlled trials, 2 cohort studies) with a total of 941 patients were included. In the VAS comparison after 6 months of follow-up, PRP combined with HA was more likely to reduce knee pain than PRP alone (standardized mean difference (SMD): $-0.31 ; 95 \%$ confidence interval (Cl): -0.55 to $-0.06 ; \mathrm{P}=0.01<0.05)$. PRP combined with HA for KOA achieved better improvement in WOMAC Function Score (SMD: $-0.32 ; 95 \% \mathrm{Cl}$ : -0.54 to -0.10 ) and WOMAC Total Score (SMD: $-0.42 ; 95 \% \mathrm{Cl}$ : -0.67 to -0.17 ) at the 12-month follow-up than the application of PRP alone. In a comparison of Lequesne Index scores at a 6-month follow-up, PRP combined with HA improved knee pain scores more than PRP alone (SMD: $-0.42 ; 95 \% \mathrm{Cl}$ : -0.67 to -0.17 ). In terms of AEs, PRP combined with HA was not significantly different from PRP or HA alone $(P>0.05)$. Conclusions Compared with intra-articular injection of PRP alone, PRP combined with HA can improve WOMAC Function Scores, WOMAC Total Score, 6month follow-up VAS ratings, and Lequesne Index scores. However, in terms of the incidence of AEs, PRP combined with HA was not significantly different from PRP or HA alone.

\section{Introduction}

Knee osteoarthritis (KOA) is a common knee degenerative disease characterized by cartilage degeneration, cartilage exfoliation, and subchondral bone hyperplasia, leading to knee pain, joint instability and functional limitations[1]. KOA severely affects patients' quality of life and is a major public health issue[2]. KOA is most common in the elderly, with an incidence of $30 \%$ to $50 \%$ in people over 65 years of age[3,4]. Epidemiological statistics show that the prevalence of KOA in Central European countries is $16.54 \%[5]$. A survey of the prevalence of KOA in 17,128 people in China showed that the overall prevalence of KOA in the Chinese population was $8.1 \%$, or approximately $5.7 \%$ in men and $10.3 \%$ in women[6]. An epidemiological survey published in PNAS shows that the incidence of KOA in the U.S. population has doubled since the mid-20th century[7]. KOA has become a high-incidence human disease and has caused a great negative impact on people's lives and work.

The Osteoarthritis Society International (OARSI) recommends conservative treatment rather than surgery as the first-line management solution for KOA, which emphasizes the importance of conservative treatment in the treatment of KOA[8]. The American College of Rheumatology (ACR) has proposed a classification in which conservative treatment includes drug treatment and non-drug treatment[9]. Nondrug treatment includes general exercise and muscle exercise, but non-drug methods often depend 
heavily on patient compliance and are difficult to control[9]. The main drug therapies include analgesics, non-steroidal anti-inflammatory drugs and corticosteroid injections[10]. Although the above drug therapies are effective to a certain degree, there are also major side effects[10,11]. In recent years, there have been an increasing number of studies on the application of intra-articular injection of platelet-rich plasma (PRP) or hyaluronic acid (HA) in the treatment of KOA. Many systematic reviews suggest that intra-articular injection of PRP can alleviate pain symptoms and improve knee function in patients with KOA compared with injection of HA alone $[10,12,13]$. However, a double-blind randomized controlled trial with a 5-year follow-up showed that the combination of HA and PRP improved pain and function in patients[14]. PRP failed to achieve any improvement in pain relief or function over HA during long-term follow-up[14].

In recent years, clinical workers have begun treating KOA with intra-articular injections of HA combined with PRP to take advantage of their synergistic therapeutic effects. The purpose of this study was to explore the efficacy and safety of PRP combined with HA in the treatment of KOA compared with intraarticular injection of PRP or HA alone, providing evidence-based evidence for the treatment of KOA.

\section{Methods}

This meta-analysis was performed strictly in accordance with the relevant requirements of the Preferred Reporting Items for Systematic Reviews and Meta-Analyses (PRISMA) Statement.

\subsection{Inclusion and exclusion criteria}

Inclusion criteria. (1) Type of study: Published randomized controlled trial (RCT) or cohort study. (2) Research subjects: People with a clear diagnosis of KOA, regardless of age, gender or nationality. (3) Intervention: The test group received intra-articular injection of PRP combined with HA, and the control group received intra-articular injection of PRP or HA. Two- or three-arm were eligible. (4) At least one of the following outcome indicators: Western Ontario and McMaster Universities Arthritis Index (WOMAC), Lequesne Index, visual analogue scale (VAS), and adverse events (AEs).

Exclusion criteria. (1) Reviews, meeting abstracts, case reports. (2) Subjects include both KOA and hip osteoarthritis. (3) The intervention did not include intra-articular injection of PRP combined with HA. (4) Duplicate publications or studies with similar data. (5) Incomplete, unclear, or obviously erroneous data that could not be resolved by contacting the authors.

\subsection{Literature retrieval strategy}

The PubMed, Cochrane Library, EMBASE and China National Knowledge Infrastructure (CNKI) databases were searched, and RCTs and cohort studies that met the inclusion criteria were included. The retrieval time was from the establishment of each database to December 2019. See Supplement 1 for the database retrieval strategies.

\subsection{Literature screening and data extraction}


Two orthopaedic surgeons conducted the literature retrieval; the preliminary and secondary screenings of the literature were performed strictly in accordance with the pre-established inclusion and exclusion criteria. The two researchers extracted the data independently, and a third researcher compared their outputs. In the event of an error or difference, the third researcher and corresponding author assisted in the judgement.

The main data extracted in this study included the title, first author, publication year, sample size, intervention measures, age, relevant items for literature quality evaluation and relevant outcome indicators of clinical efficacy and safety.

\subsection{Risk of bias assessment of included studies}

Regarding RCTs, the Cochrane risk of bias tool was used for quality evaluation[15]. The tool includes evaluation in seven areas: random sequence generation, allocation blinding, blinding of participants, blinding of outcome measures, incomplete outcome data, selective reporting, and other biases. The risk of bias in each area was judged to be low, high or unclear[16]. For cohort studies, the Newcastle-Ottawa Scale (NOS) was used for quality assessment. This scale includes three aspects: (1) selection of study groups; (2) ascertainment of exposure and outcome; and (3) group comparability. Studies with scores greater than or equal to 7 were considered to have a low risk of bias, scores of 4 to 6 indicated a moderate risk of bias, and scores less than 4 indicated a high risk of bias.

\subsection{Statistical analysis}

The relative risk (RR) was used to evaluate the effects of binary variables, the standardized mean difference (SMD) was used to evaluate the effects of continuous variables, and $95 \%$ confidence intervals (Cls) of the RR and SMD were calculated. Review Manager 5.3.5 software (Cochrane Collaboration, Oxford, UK) was used to calculate the efficacy and safety indicators and their $95 \% \mathrm{Cls}$. In addition, for homogeneous data sets, $\mathrm{P}>0.1$ and $\mathrm{I}^{2}<50 \%$ were used as the test standards. When the above two statistical conditions were met, a fixed-effects model was used for the meta-analysis because the pooled effect sizes were relatively homogenous. If one of the above standards did not conform, the homogeneity of the pooled effect size was not ideal, and a random-effects model was applied.

To quantitatively assess whether there are publication biases in different outcome indicators, this study used Stata 14.0 (STATA Corporation, Lakeway, Texas, USA) software to perform Egger's and Begg's linear regression tests on the outcome indicators included in the combined analysis of three or more studies.

\section{Results}

\subsection{Literature screening process and results}

A total of 653 related studies were obtained in the preliminary inspection, including $n=170$ from PubMed, $n=218$ from Embase, $n=128$ from the Cochrane Library, $n=37$ from CNKI, and $n=0$ from other manual 
searches. After reading the titles and abstracts and excluding irrelevant documents, a total of 27 articles remained. After excluding duplicate studies, following the inclusion criteria and exclusion criteria, this study eventually included 5 RCTs and 2 cohort studies; all 7 studies clearly stated that they had received ethical approval. The included literature included two three-arm trials, and the rest were two-arm trials, for a total of 941 patients. The literature screening process and results are shown in Figure 1. The basic information of the included literature is shown in Table 1. We systematically summarized the preparation of PRP combined with HA and the dosage, frequency, and duration of treatment of the 7 studies included. The results are shown in Supplement 2.

\subsection{Quality evaluation of the included literature}

Quality evaluation of the 5 RCTs. There are 3 studies that explicitly report the specific method of using random allocation, such as the random number table method, and 2 studies merely mention randomness and do not explain the specific method. Three papers did not explain allocation and hiding, and 1 paper did not perform allocation and hiding. The blinding risk of the participants in the blind method and the outcome index measurement process was mainly unclear risk and low risk, and no high risk was found in the literature. None of the five RCTs had missing data, selective reporting, or other risks (Figure 2 and Figure 3).

Quality evaluation of the 2 cohort studies. The NOS scores of both cohort studies were 9 , and both were low risk (Table 2).

\subsection{Meta-analysis}

\subsubsection{VAS}

A total of 3 studies reported VAS at 1 month after treatment. The heterogeneity test indicated that the homogeneity was not ideal $\left(I^{2}=97 \%, P<0.00001\right)$. A random-effects model was used for the metaanalysis. The results showed that PRP combined with HA was not significantly different from PRP alone (SMD: $-1.13,95 \% \mathrm{Cl}:-2.84$ to $0.13, \mathrm{P}=0.19>0.05$ ) (Figure 4).

A total of 2 articles reported VAS at 3 months after treatment. The heterogeneity test indicated heterogeneity $\left(I^{2}=57 \%, P=0.13\right)$, and a random-effects model was used for meta-analysis. The results showed that PRP combined with HA was not significantly different from PRP alone (SMD: $-0.36,95 \% \mathrm{Cl}$ : -0.92 to $0.20, P=0.20>0.05$ ) (Figure 4).

A total of 4 studies reported VAS at 6 months after treatment. The heterogeneity test suggested a high degree of homogeneity $\left(I^{2}=0 \%, P=1.00\right)$, and a fixed-effects model was used for meta-analysis. The results showed that the difference between PRP combined with HA compared with PRP alone was statistically significant (SMD: $-0.31,95 \% \mathrm{Cl}:-0.55$ to $-0.06, \mathrm{P}=0.01<0.05)$ (Figure 5).

\subsubsection{WOMAC Function Score}


A total of 2 studies reported the WOMAC Function Score at 12 months after treatment. The heterogeneity test showed good homogeneity $\left(I^{2}=40 \%, P=0.20\right)$, and a fixed-effects model was used for the metaanalysis. The results showed that the difference between PRP and HA compared with PRP alone was statistically significant (SMD: $-0.32,95 \% \mathrm{Cl}$ : -0.54 to $-0.10, \mathrm{P}=0.004<0.05)$ (Figure 6).

\subsubsection{WOMAC Total Score}

A total of 2 studies reported comparisons of the WOMAC Total Score at 12 months after treatment. The heterogeneity test indicated that the homogeneity was good $\left(\mathrm{I}^{2}=0 \%, \mathrm{P}=0.90\right)$, and a fixed-effects model was used for meta-analysis. The results showed that the difference between PRP combined with HA compared with PRP alone was statistically significant (SMD: $-0.42,95 \% \mathrm{Cl}:-0.67$ to $-0.17, \mathrm{P}=0.001<0.05$ ) (Figure 7).

\subsubsection{Lequesne Index}

A total of 2 studies reported Lequesne Index scores 6 months after treatment. The heterogeneity test indicated that the homogeneity was better $\left(I^{2}=15 \%, P=0.28\right)$, and a fixed-effects model was used for meta-analysis. The results showed that PRP combined with HA had significant differences compared with PRP alone (SMD: $-0.42,95 \% \mathrm{Cl}:-0.67$ to $-0.17, \mathrm{P}<0.00001$ ) (Figure 8).

\subsubsection{AEs}

A total of 5 studies reported the comparison of AEs of PRP combined with HA and PRP alone on KOA. The heterogeneity test showed that the homogeneity was good $\left(I^{2}=13 \%, P=0.33\right)$, and a fixed-effects model was used for meta-analysis. The results showed no significant difference between PRP combined with HA compared with PRP alone (RR: 0.92, 95\% Cl: 0.54 to 1.58, P=0.77) (Figure 9).

A total of 2 studies reported the comparison of AEs of PRP combined with HA and the application of HA alone in the treatment of KOA. The heterogeneity test suggested a high degree of homogeneity $\left(I^{2}=0 \%\right.$, $P=0.50)$, and a fixed-effects model was used for meta-analysis. The results showed that PRP combined with HA had no significant difference compared with the application of HA alone (RR: $0.92,95 \% \mathrm{Cl}: 0.49$ to $1.75, P=0.81$ ) (Figure 10).

\subsection{Evaluation of publication bias}

To quantitatively analyse whether there are publication biases in the relevant outcome indicators of this study, we conducted Egger's and Begg's tests on the outcome indicators combined with 3 or more studies. The results showed that there was no publication bias in the results of VAS after 1 month of treatment (Begg's test: $\operatorname{Pr} \otimes|z|=1.000 \otimes 0.05$; Egger's test: $P=0.857 \otimes 0.05)$, VAS after 6 months of treatment (Begg's test: $\operatorname{Pr} \||z|=0.734 \otimes 0.05$; Egger's test: $P=0.619 \otimes 0.05$ ), and AEs (Begg's test: $\operatorname{Pr} \otimes|z|=0.221 \otimes 0.05 ;$ Egger's test: $P=0.269 \otimes 0.05)$. The data analysis process and statistical results of publication bias are shown in

\section{Supplementary 3.}




\section{Discussion}

$\mathrm{KOA}$ is a disease that can cause lower extremity disability, reduce the quality of life of patients, and seriously affect the physical and mental health of middle-aged and elderly people[24]. With the ageing of the population, KOA will gradually become a common and frequently occurring disease, which is a major challenge that health systems in various countries need to meet[25]. The pathogenesis of knee osteoarthritis is still unclear, and there is still no continuous and effective conservative treatment[10]. For patients with KOA who are not treated well with conservative treatment, surgical treatment is mostly used, but surgical treatment is mostly used for patients with severe KOA[26]. Moreover, surgical treatment has a long recovery period, unavoidable risks of surgery and complications[27]. Therefore, it is of great significance to study new treatments for KOA. In recent years, intra-articular injection of PRP or HA for the treatment of KOA has attracted strong interest from many clinicians, and in-depth research has been conducted $[28,29]$.

PRP is extracted by centrifugation from autologous blood, and the platelet concentration can be increased nearly 10 -fold, which contains approximately 1500 proteins that can release macrophages and growth factors after activation, which is beneficial not only for removing necrotic tissue and reducing the inflammatory response but also for articular cartilage repair and regeneration [30-32]. HA is a high molecular weight polysaccharide that is an important part of synovial fluid and articular cartilage. Injecting HA in the knee joint cavity can physically lubricate the articular surface, reduce wear, and biologically nourish articular cartilage and promote the synthesis of endogenous HA delays further joint disease [33-35]. A large number of RCTs and systematic reviews of intra-articular injection of PRP or HA for KOA have been published [12, 36-38], and most studies have concluded that intra-articular injection of PRP can relieve knee pain and improve the function of patients with KOA compared with HA. Studies have shown that the combined application of PRP and HA can repair degeneration of cartilage and delay the progression of KOA[39-41]. This synergistic effect mainly changes the role of inflammatory cytokines in the process of chondrocyte degeneration through specific mediators (CD44, TGF- $\beta R$ II), thereby promoting cartilage regeneration and inhibiting the inflammatory response[42].

In our study, meta-analysis showed that there was no significant difference between PRP combined with HA and PRP for KOA at 1 month or 3 months after treatment. This outcome shows that the effects of the two intervention methods in relieving knee pain are similar at 1 month and 3 months after treatment. However, VAS at 6 months after treatment showed that PRP combined with HA could relieve pain in patients with KOA compared with intra-articular injection of PRP. PRP combined with intra-articular injection of $\mathrm{HA}$ has a unique advantage in the long-term relief of pain in patients with KOA, but the results of longer follow-up are still needed for comparison. We found that PRP combined with HA at 6 months after treatment was superior to PRP alone, which may suggest that PRP combined with HA may be a better treatment for patients with long-term knee pain in the future. In terms of the improved WOMAC Function Score and WOMAC Total Score, PRP combined with HA can improve patients' knee joint function scores and overall WOMAC scores compared with intra-articular injection of PRP. From the analysis of Lequesne Index scores, we found that PRP combined with HA can reduce Lequesne Index 
scores more than PRP alone, which showed that PRP is more effective in relieving knee pain. This systematic review compares and analyses the adverse reactions of PRP combined with HA compared with PRP and HA alone for KOA. The results showed that no significant difference was found in the incidence of adverse reactions, whether PRP, HA, or both were applied, indicating that the safety of the three treatments was not different. The meta-analysis conducted Begg's and Egger's tests on VAS after 1 month and 6 months of treatment and AEs. The results suggest that there is no publication bias, indicating that the above results are reliable.

Nevertheless, several limitations were unavoidable. First, 2 articles were non-RCTs, which may lead to heterogeneity of the combined indicators. Second, the follow-up time was short, with the longest followup period being 1 year, and the long-term efficacy and safety of PRP combined with HA could not be evaluated. This is a direction that will require special attention in later clinical research. Third, from the indicators related to WOMAC, it is found that due to the inconsistent indicators reported in the literature, the combined results of the indicators related to WOMAC Pain Score and WOMAC Stiffness Score are lacking. In later clinical studies, attention should be paid to the comprehensiveness and consistency of the outcome indicators. Fourth, there are few studies that directly compare the efficacy and safety of PRP combined with $\mathrm{HA}$ and intra-articular injection of $\mathrm{HA}$ alone. This meta-analysis failed to fully compare the efficacy of PRP combined with HA to that of HA alone.

\section{Conclusion}

The results of this study indicate that PRP combined with HA may have promising clinical effects on KOA. Based on our results, compared with intra-articular injection of PRP, PRP combined with HA can improve WOMAC Function Scores, WOMAC Total Scores, VAS ratings (after 6 months of treatment), and Lequesne Index scores. However, in terms of the incidence of AEs, PRP combined with HA was not significantly different from intra-articular injection of PRP or HA, and the safety of the three treatment regimens was similar. Because there are only a few studies included in this review, additional high-quality long-term follow-up RCTs are needed to supplement and update the relevant results of this meta-analysis.

\section{Abbreviations}

platelet-rich plasma:PRP

Hyaluronic Acid:HA

Knee Osteoarthritis:KOA

Western Ontario and McMaster Universities Arthritis Index:WOMAC

visual analog scale:VAS

adverse events:AEs 
China National Knowledge Infrastructure:CNKI

randomized controlled trail:RCT

Newcastle-Ottawa Scale:NOS

relative risk: $\mathrm{RR}$

Standard Mean Difference:SMD

\section{Declarations}

\section{Acknowledgements}

We thank American Journal Experts for linguistic assistance during the preparation of this manuscript.

\section{Authors' contributions}

Jinlong Zhao,Hetao Huang and Guihong Liang had the original idea for the study and proposed the study design. Lingfeng Zeng,Weiyi Yang and Guihong Liang conducted the literature search, screened and selected the studies initially identified.Jinlong Zhao,Hetao Huang and Guihong Liang read and evaluated the quality of the studies included.Jinlong Zhao and Hetao Huang conducted the meta-analysis.Jun Liu wrote the initial manuscript and serves as guarantor.All authors contributed to interpreting the study findings and to the final manuscript.

\section{Funding}

This work was funding by the Project of Guangdong Provincial Department of Finance (No.[2014]157, No. [2018]8), the China Postdoctoral Science Foundation (No. 2018M633036), the Medical Science Research Foundation of Guangdong Province (No. B2019091), the National Natural Science Foundation of China (No. 81873314), Key scientific research platforms and research projects of universities in Guangdong Province (No. 2018KQNCX041), and the Science and Technology Research Project of Guangdong Provincial Hospital of Chinese Medicine (No.YN2019ML08, No. YK2013B2N19, YN2015MS15).

\section{Ethics approval and consent to participate}

Not applicable.

\section{Consent for publication}

All authors agree to publish in BMC Musculoskeletal Disorders.

\section{Availability of data and material}

All data and materials are contained within the manuscript. 


\section{Competing interests}

The authors declare that they have no competing interests.

\section{References}

1.Pereira D, Peleteiro B, Araújo J, Branco J, Santos RA, Ramos E.The effect of osteoarthritis definition on prevalence and incidence estimates: a systematic review. OSTEOARTHR CARTILAGE.2011, 19(11):12701285.

2.Williams QI, Gunn AH, Beaulieu JE, Benas BC, Buley B, Callahan LF, Cantrell J, Genova AP, Golightly YM, Goode AP et al.Physical therapy vs. internet-based exercise training (PATH-IN) for patients with knee osteoarthritis: study protocol of a randomized controlled trial. BMC MUSCULOSKEL DIS.2015, 16(1):264.

3.Loeser RF.Age-Related Changes in the Musculoskeletal System and the Development of Osteoarthritis. CLIN GERIATR MED.2010, 26(3):371-386.

4.Bruyn GA, Naredo E, Damjanov N, Bachta A, Baudoin P, Hammer HB, Lamers-Karnebeek FB, Moller Parera I, Richards $\mathrm{B}$, Taylor $\mathrm{M}$ et al.An OMERACT reliability exercise of inflammatory and structural abnormalities in patients with knee osteoarthritis using ultrasound assessment. ANN RHEUM DIS.2016, 75(5):842-846.

5.Horváth G, Koroknai G, Ács B, Than P, Bellyei Á, llés T.Prevalence of radiographic primary hip and knee osteoarthritis in a representative Central European population. INT ORTHOP. 2011, 35(7):971-975.

6.Tang X, Wang S, Zhan S, Niu J, Tao K, Zhang Y, Lin J.The Prevalence of Symptomatic Knee Osteoarthritis in China: Results From the China Health and Retirement Longitudinal Study. ARTHRITIS RHEUMATOL.2016, 68(3):648-653.

7.Wallace IJ, Worthington S, Felson DT, Jurmain RD, Wren KT, Maijanen H, Woods RJ, Lieberman DE.Knee osteoarthritis has doubled in prevalence since the mid-20th century. Proceedings of the National Academy of Sciences.2017, 114(35):9332-9336.

8.Bannuru RR, Osani MC, Vaysbrot EE, Arden NK, Bennell K, Bierma-Zeinstra S, Kraus VB, Lohmander LS, Abbott $\mathrm{JH}$, Bhandari $\mathrm{M}$ et al.OARSI guidelines for the non-surgical management of knee, hip, and polyarticular osteoarthritis. Osteoarthritis Cartilage.2019, 27(11):1578-1589.

9.Guidelines AACO.Recommendations for the medical management of osteoarthritis of the hip and knee: 2000 update. American College of Rheumatology Subcommittee on Osteoarthritis Guidelines. Arthritis Rheum 2000, 43(9):1905-1915.

10. Han Y, Huang H, Pan J, Lin J, Zeng L, Liang G, Yang W, Liu J: Meta-analysis Comparing Platelet-Rich Plasma vs Hyaluronic Acid Injection in Patients with Knee Osteoarthritis. PAIN MED 2019, 20(7):14181429. 
11. Hepper CT, Halvorson JJ, Duncan ST, Gregory AJ, Dunn WR, Spindler KP: The efficacy and duration of intra-articular corticosteroid injection for knee osteoarthritis: a systematic review of level I studies. J Am Acad Orthop Surg.2009, 17(10):638-646.

12.Dai W, Zhou A, Zhang H, Zhang J. Efficacy of Platelet-Rich Plasma in the Treatment of Knee Osteoarthritis: A Meta-analysis of Randomized Controlled Trials. Arthroscopy: The Journal of Arthroscopic \& Related Surgery .2017, 33(3):659-670.

13.Chen P, Huang L, Ma Y, Zhang D, Zhang X, Zhou J, Ruan A, Wang Q.Intra-articular platelet-rich plasma injection for knee osteoarthritis: a summary of meta-analyses. J ORTHOP SURG RES.2019, 14(1):16.

14.Di Martino A, Di Matteo B, Papio T, Tentoni F, Selleri F, Cenacchi A, Kon E, Filardo G. Platelet-Rich Plasma Versus Hyaluronic Acid Injections for the Treatment of Knee Osteoarthritis: Results at 5 Years of a Double-Blind, Randomized Controlled Trial. The American Journal of Sports Medicine.2019, 47(2):347354.

15. Higgins JPT, Altman DG, Gotzsche PC, Juni P, Moher D, Oxman AD, Savovic J, Schulz KF, Weeks L, Sterne JAC.The Cochrane Collaboration's tool for assessing risk of bias in randomised trials. BMJ.2011, 343(oct18 2):d5928.

16.Feng Q, Zhou A, Zou H, Ingle S, May MT, Cai W, Cheng C, Yang Z, Tang J. Quadruple versus triple combination antiretroviral therapies for treatment naive people with HIV: systematic review and metaanalysis of randomised controlled trials. BMJ.2019:14179.

17.Abate M, Verna S, Schiavone C, Di Gregorio P, Salini V. Efficacy and safety profile of a compound composed of platelet-rich plasma and hyaluronic acid in the treatment for knee osteoarthritis (preliminary results). European Journal of Orthopaedic Surgery \& Traumatology. 2015, 25(8):1321-1326.

18.Yu W, Xu P, Huang G, Liu L.Clinical therapy of hyaluronic acid combined with platelet-rich plasma for the treatment of knee osteoarthritis. EXP THER MED.2018, 16(3):2119-2125.

19.Guo Y, Yu H, Yuan L: Treatment of knee osteoarthritis with platelet-rich plasma plus hyaluronic acid in comparison with platelet-rich plasma only. INT J CLIN EXP MED.2016, 9(6):12085-12090.

20.DING Quanwei1,LV Shuaijie, SHEN Xingchao, TONG Peijian.A prospective randomized controlled study on platelet-rich plasma (PRP) combined with sodium hyaluronate (HA) intra-articular injection in the treatment of knee osteoarthritis.Shanghai Medical \& Pharmaceutical Journal.2017, 38(05):25-28.

21.ZHAO Xin-liang.Clinical effect of sodium hyaluronate injection combined with autologous platelet rich plasma injection for knee osteoarthritis. Clinical Research and Practice.2018, 3(25):37-38.

22.Guo Yanqing,Yu Hongbo,Meng Yong,Li Guandong,Lyv Huili,Yuan Lin,Man Zhentao,Li Wei2, Sun Shui.Treatment of knee osteoarthritis with mixture of platelet-rich-plasma plus hyaluronic acid.Chinese Journal of Joint Surgery(Electronic Edition).2018, 12(06):874-878. 
23.KE Chen-rong,ZHANG Rui,XUE Ji-xin.Clinical efficacy of autologous platelet-rich plasma combined with intra-articular hyaluronic acid injection for knee osteoarthritis. Chinese Journal of General Practice.2016, 14(11):1810-1812.

24.0o WM, Liu X, Hunter DJ.Pharmacodynamics, efficacy, safety and administration of intra-articular therapies for knee osteoarthritis. EXPERT OPIN DRUG MET.2019, 15(12):1021 -1032.

25.Huétink K, Stoel BC, Watt I, Kloppenburg M, Bloem JL, Malm SH, van T Klooster R, Nelissen RGHH. Identification of factors associated with the development of knee osteoarthritis in a young to middle-aged cohort of patients with knee complaints. CLIN RHEUMATOL 2015, 34(10):1769-1779.

26.Charlesworth J, Fitzpatrick J, Perera NKP, Orchard J.Osteoarthritis- a systematic review of long-term safety implications for osteoarthritis of the knee. BMC MUSCULOSKEL DIS.2019, 20(1):151.

27.Kanchanatawan W, Arirachakaran A, Chaijenkij K, Prasathaporn N, Boonard M, Piyapittayanun P, Kongtharvonskul J.Short-term outcomes of platelet-rich plasma injection for treatment of osteoarthritis of the knee. Knee Surgery, Sports Traumatology, Arthroscopy.2016, 24(5):1665-1677.

28.Laudy ABM, Bakker EWP, Rekers M, Moen MH. Efficacy of platelet-rich plasma injections in osteoarthritis of the knee: a systematic review and meta-analysis. BRIT J SPORT MED.2015, 49(10):657672.

29.Smelter E, Hochberg MC. New treatments for osteoarthritis. CURR OPIN RHEUMATOL.2013, 25(3):310316.

30.Simental-Mendía MA, Vílchez-Cavazos JF, Martínez-Rodríguez HG.El plasma rico en plaquetas en osteoartrosis de rodilla: una alternativa de tratamiento. Artículo de revisión. Cirugía y Cirujanos.2015, 83(4):352-358.

31.Bennell KL, Hunter DJ, Paterson KL. Platelet-Rich Plasma for the Management of Hip and Knee Osteoarthritis. CURR RHEUMATOL REP.2017, 19(5):24.

32.Say F, Gurler D, Yener K, Bulbul M, Malkoc M: Platelet-rich plasma injection is more effective than hyaluronic acid in the treatment of knee osteoarthritis. Acta Chir Orthop Traumatol Cech 2013, 80(4):278283.

33.Duymus TM, Mutlu S, Dernek B, Komur B, Aydogmus S, Kesiktas FN.Choice of intra-articular injection in treatment of knee osteoarthritis: platelet-rich plasma, hyaluronic acid or ozone options. Knee Surgery, Sports Traumatology, Arthroscopy.2017, 25(2):485-492.

34.Richette P.Hyaluronic acid: Still useful in knee osteoarthritis? JOINT BONE SPINE.2017, 84(6):655-656.

35.Maheu E, Bannuru RR, Herrero-Beaumont G, Allali F, Bard H, Migliore A.Why we should definitely include intra-articular hyaluronic acid as a therapeutic option in the management of knee osteoarthritis: 
Results of an extensive critical literature review. SEMIN ARTHRITIS RHEU. 2019, 48(4):563-572.

36.Shen L, Yuan T, Chen S, Xie X, Zhang C.The temporal effect of platelet-rich plasma on pain and physical function in the treatment of knee osteoarthritis: systematic review and meta-analysis of randomized controlled trials. J ORTHOP SURG RES.2017, 12(1):16.

37.Al-Moraissi EA, Wolford LM, Ellis E, Neff A.The hierarchy of different treatments for arthrogenous temporomandibular disorders: A network meta-analysis of randomized clinical trials. J CRANIO MAXILL SURG.2019,48(1):9-23.

38.Wu Q, Luo X, Xiong Y, Liu G, Wang J, Chen X, Mi B. Platelet-rich plasma versus hyaluronic acid in knee osteoarthritis: A meta-analysis with the consistent ratio of injection. J ORTHOP SURG-HONG K.2020, 28(1):920548254.

39.Lana JF, Weglein A, Sampson SE, Vicente EF, Huber SC, Souza CV, Ambach MA, Vincent H, UrbanPaffaro A, Onodera $\mathrm{CM}$ et al.Randomized controlled trial comparing hyaluronic acid, platelet-rich plasma and the combination of both in the treatment of mild and moderate osteoarthritis of the knee. $J$ Stem Cells Regen Med.2016, 12(2):69-78.

40.C S, G P, S F, M F, K K, K R, H W, H A, N S, K J.Intra-articular Hyaluronic Acid (HA) and Platelet Rich Plasma (PRP) injection versus Hyaluronic acid (HA) injection alone in Patients with Grade III and IV Knee Osteoarthritis (OA): A Retrospective Study on Functional Outcome. Malaysian Orthopaedic Journal.2016, 10(2):35-40.

41.Patel S, Dhillon MS, Bansal T.Randomized controlled trial comparing hyaluronic acid, platelet-rich plasma and the combination of both in the treatment of mild and moderate osteoarthritis of the kneeLetter to the Editor \& Author Response. Journal of stem cells \& regenerative medicine.2017, 13(2):80.

42.Andia I, Abate M.Knee osteoarthritis: hyaluronic acid, platelet-rich plasma or both in association? EXPERT OPIN BIOL TH.2014, 14(5):635-649.

\section{Figures}



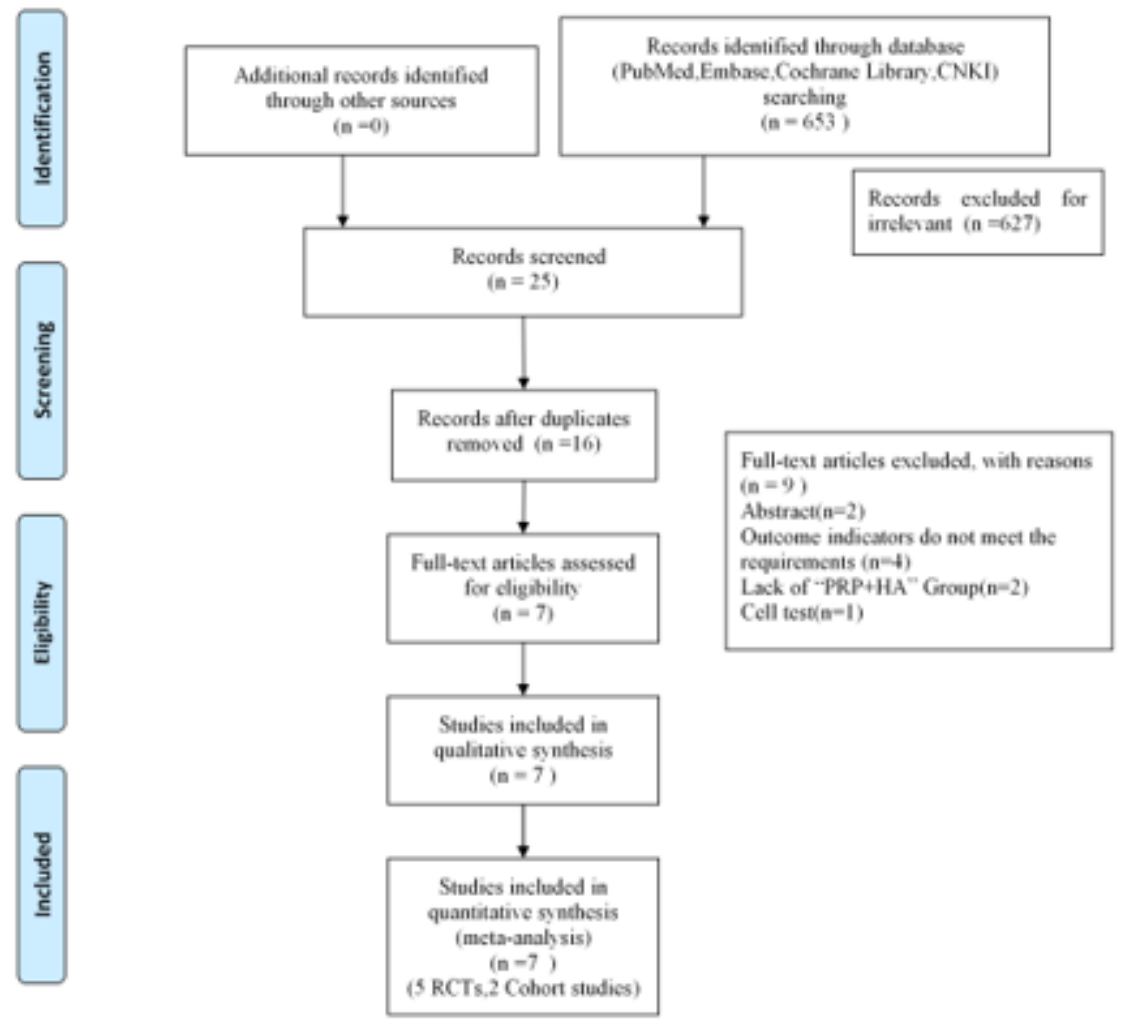

Figure 1

Flow diagram of study selection.

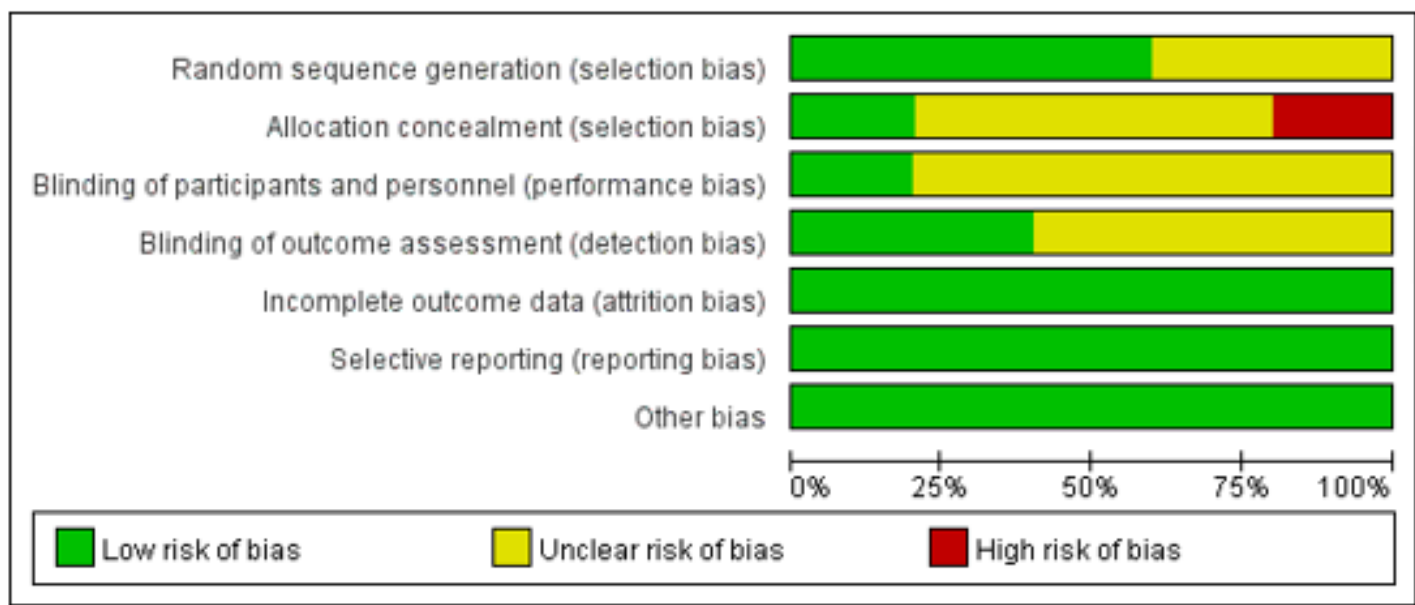

\section{Figure 2}

\section{Risk of bias assessment.}




\begin{tabular}{|c|c|c|c|c|c|}
\hline 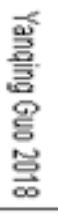 & $\begin{array}{l}\text { 즐 } \\
\text { 言 } \\
\text { 总 } \\
\text { 言 } \\
\text { 总 } \\
\end{array}$ & 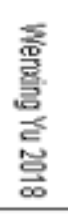 & $\begin{array}{l}\text { 을 } \\
\text { 言 } \\
\text { 言. } \\
\text { 号 } \\
\text { 总 } \\
\text { 总 }\end{array}$ & 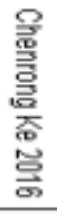 & \\
\hline$\sim$ & + & + & + & $\omega$ & Random sequence generation (selection bias) \\
\hline$\sim$ & $(1)$ & + & $\sim$ & $\sim$ & Allocation concealment (selection bias) \\
\hline$\sim$ & $\sim$ & + & $\sim$ & $\sim$ & Blinding of participants and personnel (performance bias) \\
\hline$\sim$ & $\omega$ & + & + & $\sim$ & Blinding of outcome assessment (detection bias) \\
\hline+ & + & + & + & + & Incomplete outcome data (attrition bias) \\
\hline+ & + & + & + & + & Selective reporting (reporting bias) \\
\hline+ & + & + & + & + & Other bias \\
\hline
\end{tabular}

\section{Figure 3}

\section{Risk of bias summary}

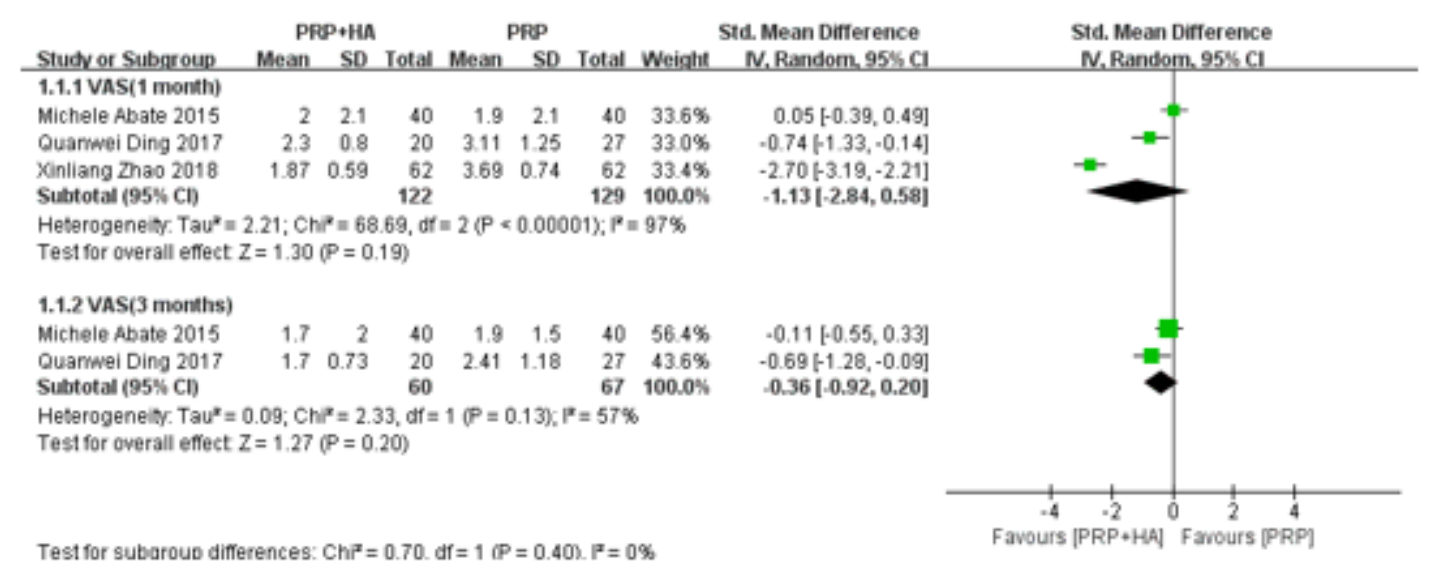

\section{Figure 4}

Forest plot and meta-analysis of VAS score( 1 and 3 months)

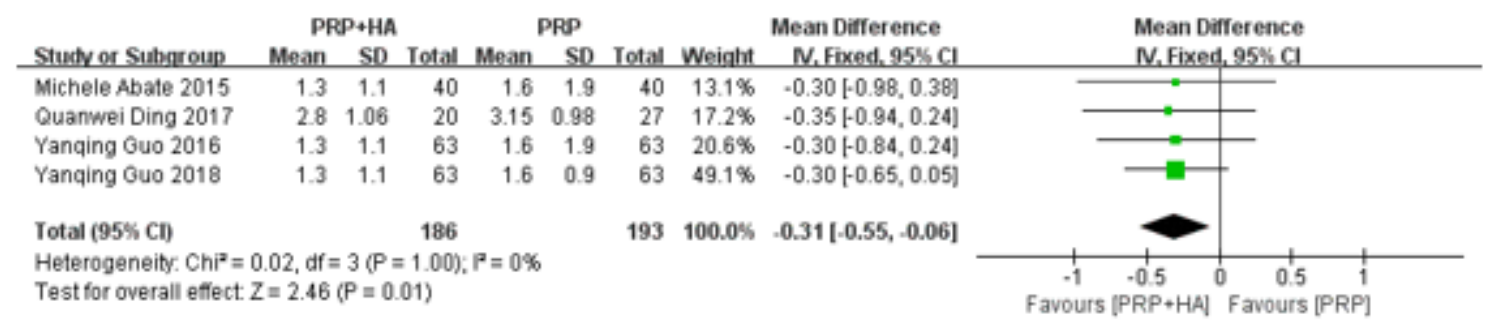

\section{Figure 5}

Forest plot and meta-analysis of VAS score(6 months) 


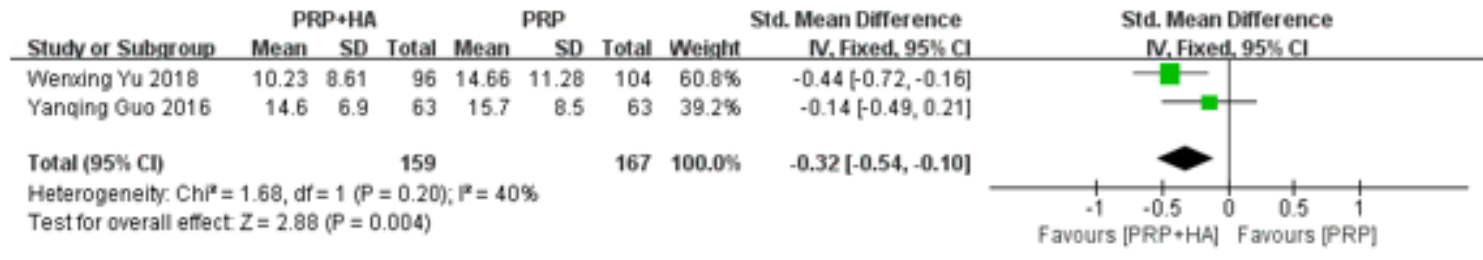

\section{Figure 6}

Forest plot and meta-analysis of WOMAC Function Score

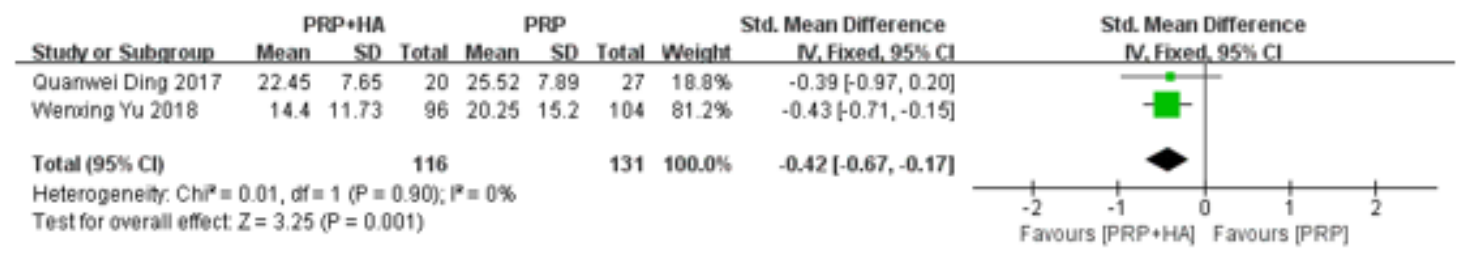

\section{Figure 7}

Forest plot and meta-analysis of WOMAC Total Score

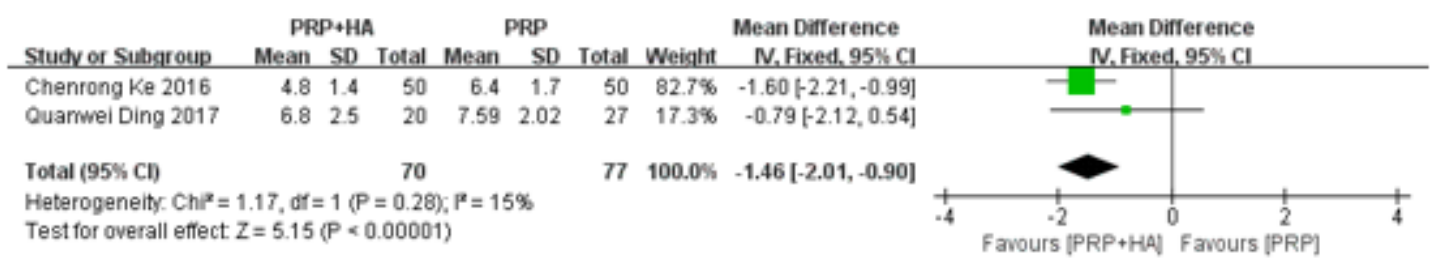

\section{Figure 8}

Forest plot and meta-analysis of Lequesne Index scores

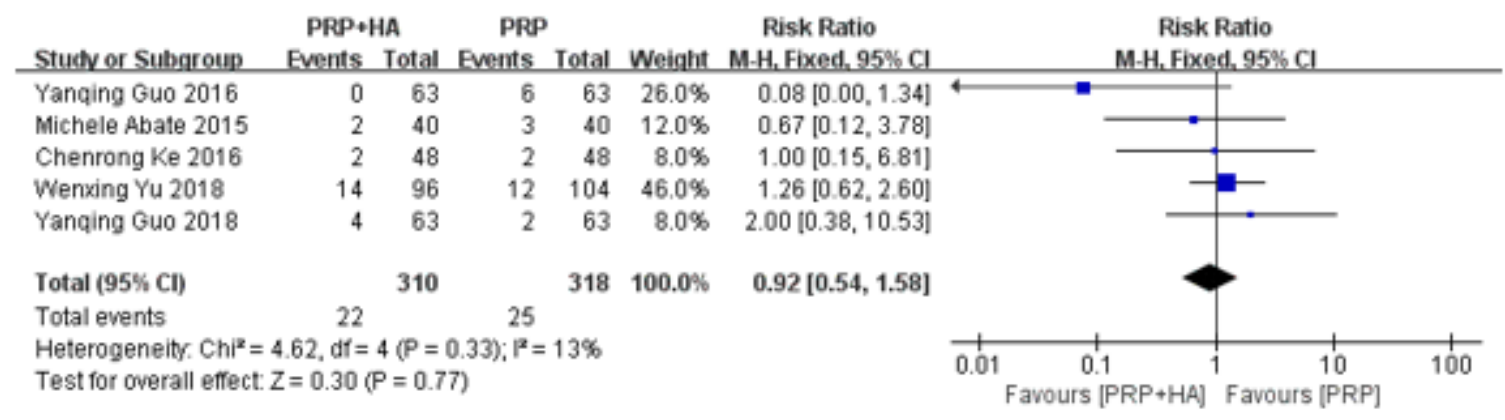

\section{Figure 9}

Forest plot and meta-analysis of Adverse events(PRP+HA VS PRP) 


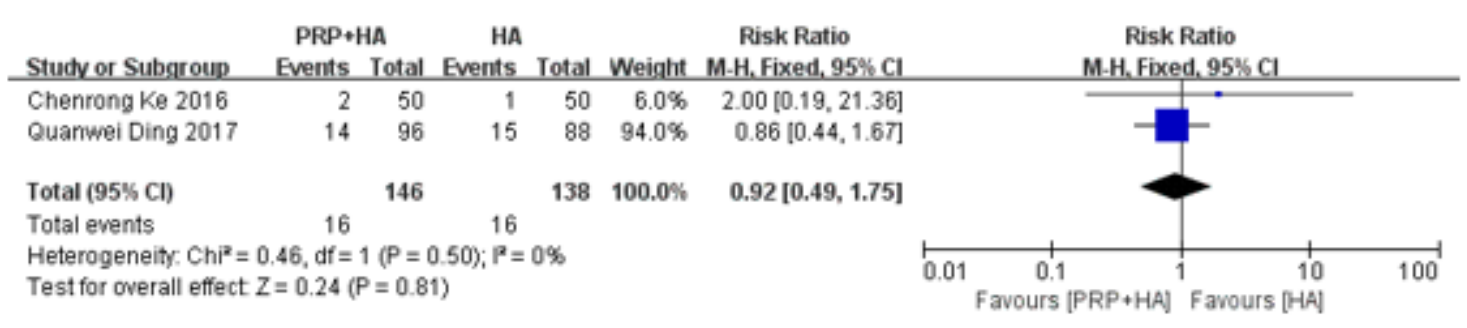

\section{Figure 10}

Forest plot and meta-analysis of Adverse events(PRP+HA VS HA)

\section{Supplementary Files}

This is a list of supplementary files associated with this preprint. Click to download.

- Supplement3PublicationBiasEggerandBeggertest.docx

- Supplement1Retrievalstrategy.docx

- Supplement2.docx

- PRISMA2009checklist.doc 